\title{
THROUGHPUT ANALYSIS IN WIDEBAND MIMO BROADCAST CHANNELS WITH PARTIAL FEEDBACK
}

\author{
Maralle J. Fakhereddin, Masoud Sharif and Babak Hassibi \\ Department of Electrical Engineering, MC 136-93 \\ California Institute of Technology, Pasadena, CA 91125 \\ Email: \{maralle, masoud, hassibi\}@systems.caltech.edu
}

\begin{abstract}
It has been recently shown that opportunistic transmit beamforming using partial channel state information (CSI) achieves the same throughput scaling obtained from dirty paper coding for a broadcast channel with fixed number of transmit antennas and many receivers [1]. In this paper, we study the generalization of this scheme to wideband broadcast channels. By using orthogonal frequency division multiplexing, an $L$-tap wideband channel can be decomposed to $N$ parallel narrowband channels, where $N$ is larger than $L$. Neighboring subchannels are therefore highly correlated, and it is intuitive to say that each group of neighboring subchannels (forming a cluster) can be characterized by one channel quality. We show in this paper that users need only feedback the best signal-to-noise-plus-interference ratio at the center of each cluster. Our results indicate that for cluster size of order $\frac{N}{\bar{L} \sqrt{\log K}}$, where $K$ is the number of users, this feedback scheme maintains the same throughput scaling as when full CSI is known. Simulation results show that larger cluster sizes $\left(\frac{N}{2 L}\right)$ can also be implemented for a small throughput hit.
\end{abstract}

\section{INTRODUCTION}

There has been growing interest in the study of the capacity region of multiple-input muItiple-output (MIMO) broadcast channels $[2,3,4]$. Recently, it has been shown that dirty paper coding achieves the capacity region of the Gaussian MIMO broadcast channel [5]. This scheme assumes perfect channel state information (CSI) at the transmitter, and achieves throughput that scales linearly with the number of transmit antennas [6]. However, full channel knowledge is not always attainable or practical. It was therefore suggested in [1] that, using $M$ random beams and partial feedback, opportunistic beamforming is performed at the trans-

This work was supported in part by the National Science Foundation under grant no. CCR-0133818, by the office of Naval Research under grant no. N00014-02-1-0578, and by Caltech's Lee Center for Advanced Networking. mitter. Each user (receiver) need only feedback its maximum signal-to-noise-plus-interference ratio (SINR) and the index of the transmitting antenna at which this maximum value exists. This scheme requires less feedback and is computationally feasible, still it achieves the linear scaling in throughput that is achieved when full CSI is available. This has been shown for narrowband broadcast channels with a fixed number of transmit antennas and large number of users, which is a typical setting in practical cellular systems.

We investigate the generalization of this scheme to an $L$-tap wideband broadcast channel (with independent and identically distributed (i.i.d.) taps). Wideband channels are desireable due to the increased need to drive bit rates higher. Using orthogonal frequency division multiplexing (OFDM), an $L$-tap wideband channel can be decomposed to $N$ parallel subchannels. However, neighboring subchannels are highly correlated (as $N$ is bigger than $L$ ), and therefore with high probability they have similar channel qualities. Recently, it has been suggested in [7] that neighboring subchannels are grouped in a cluster. Then each user need only feedback the signal-to-noise ratio (SNR) values and indices of its strongest clusters to the transmitter. Based on this opportunistic reduced feedback, the transmitter sends one beam to the user with the highest SNR value per cluster. Using simulation results, [7] shows that this scheme can be implemented without significantly sacrificing performance. There have also been recent publications that looked at variations of this problem, for example [8, 9] among others.

In this paper, we look into the effects of partial feedback on the throughput of wideband broadcast channels. More specifically we address the question of how much feedback is necessary in order to maintain the same throughput scaling as when full CSI is available. The amount of feedback is inversely proportional to the cluster size, therefore this question also addresses the issue of cluster size. Through analysis and simulations of a wideband broadcast channel with $M$ transmit antennas and $K$ single-antenna users, we prove that for cluster size of order $\frac{N}{L \sqrt{\log K}}$, each user need only feedback its best SINR value at the center subcarrier 
of each cluster in order for the transmitter to perform opportunistic beamforming (by constructing $M$ random beams rather than a single beam). With this cluster size, this scheme achieves the same throughput scaling as when full CSI is available at the transmitter with less complexity and feedback. Our simulation results show that larger cluster size $\left(\frac{N}{2 L}\right)$ can also be implemented for a small loss in throughput.

\section{PROBLEM FORMULATION}

We start with a discrete-time baseband model of the channel. At time $t$ and sampling at multiples of $\frac{1}{W}$, where $W$ is the bandwidth of the input signal, the input-output relationship can be written as

$$
y[t]=\sum_{l=0}^{L-1} h_{I} x[t-t]+w[t]
$$

where $y$ is the sampled output at time $t, x$ is the sampled input transmitted at time $t-l$ and $w$ is additive white noise. $h_{l}$ is the $l^{t h}$ complex channel filter tap, where the number of taps $L$ is dictated by the delay spread of the channel $T_{d}$ $\left(L=T_{d} \times W\right)$, and the coherence bandwidth $W_{c}=\frac{1}{2 T_{d}}$. In practice, wideband channels can be represented by 4 or 5 channel taps [10]. We assume i.i.d channel taps, and form a vector of the circularly symmetric complex Gaussian random variables $H=\left[h_{0} h_{1} \ldots h_{L-1}\right]^{T}$. Using OFDM, data symbols modulate $N$ subcarriers separated by $\frac{W}{N}$. The discrete Fourier transform of the $L$-tap channel gives the frequency response at frequency $q$ as follows,

$$
H(q)=\sum_{l=0}^{L-1} h_{l} e^{-j 2 \pi q \frac{l}{N}}, q=1, \ldots, N .
$$

In matrix form, (2) can be written as $H(q)=V_{q} H$ where

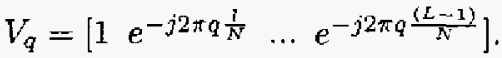

The autocomelation function depends solely on the frequency separation between subcarriers. It is therefore clear that a separation of $W_{c}$ is required between subcarriers in order to de-correlate. Since the width of each subchannel is $\frac{W}{N}$, it is straightforward to show that there are roughly $\frac{N}{2 L}$ neighboring subchannels that are highly correlated [10]. For this reason, we group neighboring subchannels in a cluster [7], and adopt a transmission scheme on each subchannel that requires partial feedback while maximizing the throughput [1].

We construct $M$ random orthonormal beams $\phi_{m}(M \times 1)$ for $m=1, \ldots, M$, and at time $t$, the $m^{\text {th }}$ vector is multiplied by the transmit symbol $s_{m}^{q}$. The transmitted signal on subchannel $q$ is then,

$$
S_{q}(t)=\sum_{m=1}^{M} s_{m}^{q}(t) \phi_{m}(t)
$$

The $L \times 1$ vector of channel gains at the $m^{\text {th }}$ beam is denoted by $H_{m}$. We assume that the $k^{\text {th }}$ receiver knows $H_{m}^{k}$ for $m=1, \ldots, M$ (can be readily available through training), and can therefore compute the following $M \operatorname{SINR}_{\mathrm{q}, \mathrm{m}}^{\mathrm{k}}$ values by assuming that $s_{m}$ is the desired signal and the other $s_{i}$ signals are interference as follows,

$\operatorname{SINR}_{\mathrm{q}, \mathrm{m}}^{\mathrm{k}}=\frac{\mathrm{H}_{\mathrm{m}}^{\mathrm{k}^{*}} \mathrm{~V}_{\mathrm{q}}^{*} \mathrm{~V}_{\mathrm{q}} \mathrm{H}_{\mathrm{m}}^{\mathrm{k}}}{\frac{1}{\rho}+\sum_{\mathrm{i}=1, \mathrm{i} \neq \mathrm{m}}^{\mathrm{M}} \mathrm{H}_{\mathrm{i}}^{\mathrm{k}^{*}} \mathrm{~V}_{\mathrm{q}}^{*} \mathrm{~V}_{\mathrm{q}} \mathrm{H}_{\mathrm{i}}^{\mathrm{k}}}, \mathrm{m}=1, \ldots, \mathrm{M}$.

$\rho$ in (4) is the SNR for all the users, assuming a homogeneous network.

We consider a feedback scheme that requires one feedback per cluster for each user. We propose that each user feeds back the best SINR value at the center subcarrier of each cluster. This feedback can be expressed as (in addition to cluster and transmit antenna indices), $\max _{1 \leq m \leq M} \operatorname{SINR}_{\mathrm{q}, \mathrm{m}}^{\mathrm{k}}$, where $q$ here is the center subcarrier in the cluster. The transmitter assigns the $m^{\text {th }}$ beam to the user with the highest SINR; $\max _{1 \leq k \leq K} \operatorname{SINR}_{\mathrm{q}, \mathrm{m}}^{\mathrm{k}}$. The throughput of each subchannel is estimated by,

$$
R_{q}=E\left\{\sum_{i=1}^{M} \log \left(1+\max _{1 \leq k \leq K} \operatorname{SINR}_{\mathrm{q}, \mathrm{m}}^{\mathrm{k}}\right)\right\},
$$

and the throughput of the system is $R=\sum_{q=1}^{N} R_{q}$. Note that for $K \rightarrow \infty,(5)$ is a tight estimate [1].

In order to further reduce the amount of feedback, we further assume that $\operatorname{SINR}_{q_{, m}}^{\mathbf{k}} \geq \gamma$ where $\gamma$ is a specified threshold of order $\log K$. We are able to set this threshold since we know that $\max _{1 \leq k \leq K} \mathrm{SINR}_{\mathrm{q}, \mathrm{m}}^{\mathrm{k}}$ behaves like $(\log K)$ with high probability [1], and therefore we restrict feedback of only SINR values $\geq \gamma$. The amount of feedback from each user then reduces to the number of clusters $x$ $\operatorname{Pr}\left(\operatorname{SINR}_{\mathrm{q}}>\gamma\right)$.

In summary, we tackle two main questions in this paper The first is concerned with justifying that the channel quality at the center subcarrier is indeed a valid representative of the channel qualities at (most of) the subcarriers in a cluster. And the second question is related to how big clusters should be (and subsequently how much feedback should we have) in order to maintain the same throughput scaling as when full CSI is available. We will answer these questions in the following section.

\section{FEEDBACK SCHEME ANALYSIS}

Due to correlations between adjacent subchannels, their corresponding adjacent SINR values are also correlated. This means that it should be satisfactory to have much less feedback than $N$ per user in order to obtain sufficient channel 


$$
\begin{aligned}
\Phi(P, Q) & =\frac{e^{\frac{-1}{\rho}\left(j w_{p} \gamma_{p}+j w_{q} \gamma_{q}\right)}}{M^{L M} \operatorname{det}\left[\frac{I_{L}}{M}-\left(j w_{p} V_{p}^{*} V_{p}+j w_{q} V_{q}^{*} V_{q}\right)\right] \operatorname{det}\left[\frac{I_{L}}{M}+\left(j w_{p} \gamma_{p} V_{p}^{*} V_{p}+j w_{q} \gamma_{q} V_{q}^{*} V_{q}\right)\right]^{M i-1}} \\
& \left.=\overline{\left[1-\left(j w_{p}+j\right.\right.} \overrightarrow{\left.w_{q}\right)} \overline{L M-w_{p}} \frac{e^{\frac{-1}{\rho}\left(j w_{p} \gamma_{p}+j w_{q} \gamma_{q}\right)}}{\left.\bar{L}\left(L^{2}-f\right) M^{2}\right]}\right]\left[1+\left(j w_{p} \gamma_{p}+j w_{q} \gamma_{p}\right) L M-w_{p} w_{q} \gamma_{p} \gamma_{q}\left(L^{2}-f\right) M^{2}\right]^{M-1}
\end{aligned}
$$

Table 1. Joint Characteristic Function

knowledge at the transmitter. The fewer the number of clusters is, the lesser feedback the transmitter will need. However, the size of the cluster influences how correlated the subchannels are. Small cluster sizes guarantee that the subchannels are highly correlated and that their channel quality is almost the same, therefore resulting in higher throughput. Whereas.wider cluster sizes will include less correlated subchannels especially toward the edges of the cluster, which will subsequently decrease the throughput. We consider that channel quality at the center subcarrier as a valid representative (and therefore sufficient feedback) for the quality of the channels at (most of) subcarriers in a cluster.

In fact we know that the best SINR over all users behaves like $\log K$. As the cluster size increases, the likelihood of the edge subcarrier (the farthest) being of order $\log K$ decreases. In this section, we show that if the size of the cluster is of order $\frac{N}{L \sqrt{\log K}}$, then with high probability the SINR at the edge subcarrier is also of order $\log K$. In other words, we show that the following probability,

$$
\operatorname{Pr}\left\{\operatorname{SINR}_{\mathrm{p}, \mathrm{m}}^{\mathrm{k}} \geq \gamma_{\mathrm{p}} \mid \operatorname{SINR}_{\mathrm{q}, \mathrm{m}}^{\mathrm{k}} \geq \gamma_{q}\right\}
$$

is close to one when $p-q=\frac{N}{L \sqrt{\log K}}^{1}, M$ is fixed and $K$ increasing, $\gamma_{q}=\alpha_{q} \log K, \gamma_{p}=\alpha_{p} \log K$ and $\alpha_{q}>\alpha_{p}$.

By making the following variable definitions,

$$
\begin{aligned}
& P=H_{m}^{k^{*}} V_{p}^{*} V_{p} H_{m}^{k}-\gamma_{p} \sum_{i=1, i \neq m}^{M} H_{i}^{k^{*}} V_{p}^{*} V_{p} H_{i}^{k}-\frac{\gamma_{p}}{\rho} \\
& Q=H_{m}^{k^{*}} V_{q}^{*} V_{q} H_{m}^{k}-\gamma_{q} \sum_{i=1, i \neq m}^{M} H_{i}^{k^{*}} V_{q}^{*} V_{q} H_{i}^{k}-\frac{\gamma_{q}}{\rho},
\end{aligned}
$$

(6) can be rewritten as

$$
\operatorname{Pr}\{P \geq 0 \mid Q \geq 0\}=\frac{\operatorname{Pr}\{P \geq 0, Q \geq 0\}}{\operatorname{Pr}\{Q \geq 0\}}
$$

In order to calculate (7), we start by evaluationg the joint probability. Using properties of the unit function, this joint

\footnotetext{
${ }^{1}$ In fact, in our analysis, we equivalently assume that the correlation coefficient of $p$ and $q$ is $1-o\left(\frac{1}{\log K}\right)$
}

probability can be written as,

$$
\begin{aligned}
\operatorname{Pr}\{\mathrm{Q} \geq 0, \mathrm{P} \geq 0\}=\frac{1}{4} & +\frac{1}{4 \pi} \int \frac{\Phi(P)}{j w_{p}} d w_{p} \\
& +\frac{1}{4 \pi} \int \frac{\Phi(Q)}{j w_{q}} d w_{q} \\
& +\frac{1}{4 \pi^{2}} \iint \frac{\Phi\left(P_{i} Q\right)}{j w_{p} j w_{q}} d w_{p} d w_{q}
\end{aligned}
$$

where $\Phi$ stands for the characteristic function and the integration limits are from $-\infty$ to $\infty$. Note that the marginal terms (second and third terms in (8)) can be easily evaluated from the marginal distribution of SINR [1], and we can show that

$$
\begin{aligned}
\frac{1}{4 \pi} \int \frac{\Phi(Q)}{j w_{q}} d w_{q} & =-\frac{1}{4}-\frac{1}{2} \frac{e^{-\frac{\gamma_{q}}{\rho L M}}}{\left(1+\gamma_{q}\right)^{M-1}} \\
\frac{1}{4 \pi} \int \frac{\Phi(P)}{j w_{p}} d w_{p} & =-\frac{1}{4}-\frac{1}{2} \frac{e^{-\frac{\gamma_{p}}{L L M}}}{\left(1+\gamma_{p}\right)^{M-1}} .
\end{aligned}
$$

However, these terms cancel out with terms that appear during the evaluation of the double integral in the fourth term of (8). Therefore, the main analysis of the joint probability lies in evaluating the joint characteristic funtion and the result of the double integral in (8).

\subsection{Joint Characteristic Function}

The joint characteristic function of $P$ and $Q$ is described as $\Phi(P, Q)=E\left(e^{j u_{p} P+j w_{q} Q}\right)$. We assume that the transmit antennas are far enough from each other to assume independent channels, and use properties of the joint pdf of Gaussian random variables [11] in order to obtain the result for the joint characteristic function in closed form as indicated in table 1, where $f=\frac{\sin ^{2}\left(\pi(p-q) \frac{L}{N}\right)}{\sin ^{2}\left(\pi(p-q) \frac{1}{N}\right)}$.

Note that when $p-q=\frac{N}{L}, f=0$ which results in $P$ and $Q$ being independent. Also if we assume that the cluster size is 1 subcarrier, and therefore $p=q,\left(L^{2}-f\right)=0$, and the joint characteristic function reduces to a marginal function as in (9).

\subsection{Complex Analysis}

In order to evaluate (8), we need to obtain the double integral in the fourth term. Substituting $j w_{p}$ with $s_{p}$ and 


$$
\begin{aligned}
& \int \frac{-j}{2 \pi}-\underbrace{[1}_{s_{q}\left(L M s_{q}-1\right)}+L M\left(\gamma_{q} s_{q}+\gamma_{p}\left(\frac{L M s_{q}-1}{s_{q} M^{2}\left(L^{2}-f\right)-L M}\right)\right)+\left(\frac{L M s_{q}-1}{s_{q} M^{2}\left(L^{2}-f\right)-L M}\right) s_{q} \gamma_{p} \gamma_{q} M^{2}\left(L^{2}-f\right)]^{\frac{-\gamma_{q} s_{q}}{p}} e^{\frac{-\gamma_{p}}{p} \frac{\left(L M s_{q}-1\right)}{s_{q} M^{2}\left(L^{2}-f\right)-L M}} d s_{q}
\end{aligned}
$$

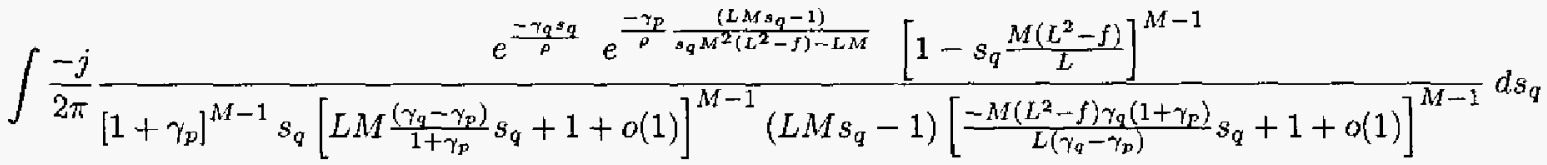

$j w_{q}$ with $s_{q}$, we perform contour integration where $s_{p}$ and $s_{q}$ go from $-\infty$ to $\infty$ on the complex axis, and the contour's direction is clock-wise. First, we integrate with respect to $s_{p}$ using Cauchy integral formula [12]. The region where $s_{p}=0$ results in a constant term that cancels out, therefore the only pole that we consider is a simple pole $s_{p}=\frac{L M s_{q}-1}{s_{q} M^{2}\left(L^{2}-f\right)-L M}$. The double integral then reduces to a single integral in terms of $s_{q}$ as shown in (10). Solving this second integral is more involved than solving the first integral. In order to proceed, we need to obtain the roots of the polynomial (raised to the power $M-1$ ).

\subsection{Asymptotic Analysis}

For the rest of the analysis, we assume that the correlation coefficient of $p$ and $q$ is $1-o\left(\frac{1}{\log K}\right)$ as $K$ increases. This implies that $\left(L^{2}-f\right) \rightarrow 0$, or equivalently that $p-q=$ $\frac{N}{L \sqrt{\log } \bar{K}}$. We also assume that the threshold for the SINR at the center subcarrier $\gamma_{q}$ behaves like $\alpha_{q} \log K$, while the edge subcarrier's threshold $\gamma_{p}$ behaves like $\alpha_{p} \log K$ where $\alpha_{q}$ and $\alpha_{p}$ are constants and $\alpha_{q}>\alpha_{p}$. We solve for the roots of the polynomial in (10) and obtain (11). Now we have two poles inside contour $s_{q}$. The first pole $s_{q}=\frac{1}{L} \frac{1}{M}$ is simple, and the second $s_{q}=\frac{L\left(\gamma_{q}-\gamma_{p}\right)}{M\left(L^{2}-f\right) \gamma_{q}\left(1+\gamma_{p}\right)}$ is of order $M-1$. In order to use Cauchy integral formula [12], we expand the exponential as follows,

$e^{\frac{-\gamma_{p}}{\rho} \frac{\left(L M s_{q}-1\right)}{s_{q} M^{2}\left(L^{2}-f\right)-L M}}=\sum_{a=0}^{\infty} \frac{\left(\frac{-\gamma_{p}}{\rho}\right)^{\alpha}}{a !}\left[\frac{L M s_{q}-1}{s_{q} M^{2}\left(L^{2}-f\right)-L M}\right]^{a}$

and divide the summation into three regions. The first region is when $a=0$, the second is when $a$ is finite ( $0<$ $a \leq M-1$ ) and the last is when $a$ goes to infinity. For the simplest case when $a=0$, we first perform partial fraction expansion on the two poles inside contour $s_{Q}$, and follow Cauchy integral formula at each pole to find the following result,

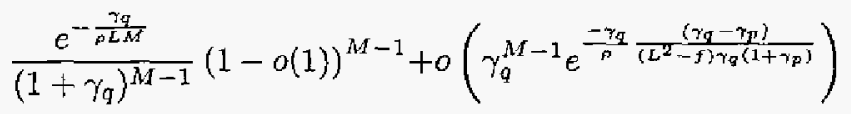

Following in the same fashion, we solve (11) for the finite and infinite $a$ regions ${ }^{2}$, and after simplifications we obtain

\footnotetext{
${ }^{2}$ Both regions result in terms that go to 0 as $K \rightarrow \infty$. This is expected since the poles in the two regions inversely depend on $\left(L^{2}-f\right)$, and there-
}

the result of (8) as,

$$
\operatorname{Pr}\{\mathrm{P} \geq 0, \mathrm{Q} \geq 0\}=\frac{\mathrm{e}^{-\frac{\gamma_{\mathrm{q}}}{\rho \mathrm{ML}}}}{\left(1+\gamma_{\mathrm{q}}\right)^{\mathrm{M}_{\mathrm{L}}-1}}[1-\mathrm{o}(1)]^{\mathrm{M}-1}
$$

and the conditional probability as,

$$
\operatorname{Pr}\{\mathrm{P} \geq 0 \mid \mathrm{Q} \geq 0\}=1-\mathrm{o}(1)
$$

This proves that when SINR at the center subcarrier is of order $\log K$, and if we keep our cluster size as $\frac{N}{L \sqrt{\log K}}$, then with high probability SINR at the edge subcarrier is also of order $\log K$ for large $K$. This implies that all the subcarriers that are chosen for transmission have SINRs of order $\log K$. Therefore, we obtain throughput scaling as $M N \log \log K$ using our scheme with much less complexity and feedback. In fact, each user need only feedback its best SINR at the center of each cluster only if it is greater than some threshold $\gamma$. This threshold comes from the fact that we already know the order of the best SINR. This reduces the feedback to $L \sqrt{\log K} \times \operatorname{Pr}\left(\mathrm{SINR}_{\mathrm{g}, \mathrm{m}} \geq \gamma\right)$ per user. Our simulation results show that wider cluster size is possible. This implies that $L^{2}-f$ is a constant (not tending to zero), which we do not analyze in this paper.

\section{SIMULATION RESULTS}

To further verify our results, we conduct Monte Carlo simulations for a wideband broadcast channel with a three-antenna transmitter, single-antenna users, and 256 subcarriers. In this section, we discuss two simulation results. Figure (1) shows the throughput as a function of number of users for various cluster sizes. Due to subchannel correlation which depends on frequency separation, and due to the relationship between frequency coherence and channel delay spread, it is intuitive that cluster width of $\frac{N}{2 L}$ achieves good performance. Indeed, figure (1) shows that cluster size $\frac{N}{2 L}$ results in a small throughput loss, and we obtain throughput close to that when cluster size is 1 subcarrier (in which case the transmitter has information at each subchannel). However,

fore go to $\infty$ as $K \rightarrow \infty$. From the definition of our cluster, we predict that their residues are very close to 0 . 


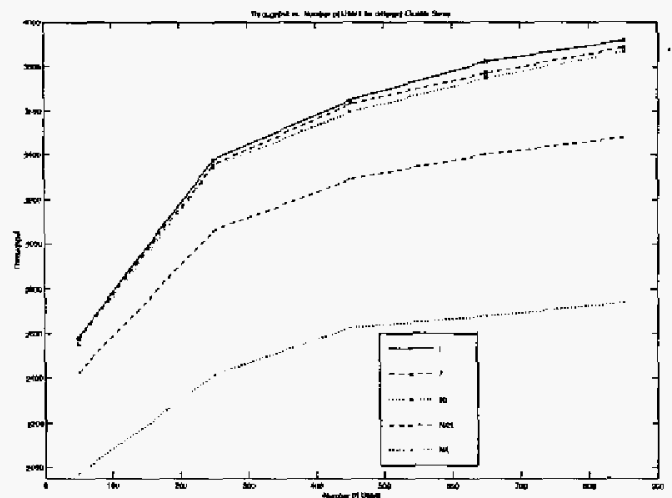

Fig. 1. Throughput vs. Number of users for different Cluster $\operatorname{sizes}(\mathrm{N}=256, \mathrm{~L}=4, \mathrm{M}=3, \mathrm{SNR}=10)$.

as suggested by our analysis, figure (1) indicates that cluster sizes of 7 and 10 result in throughput scaling as with full CSI. This demonstrates that when we stay in the region of the highest SINR values (those that behave like $\log K$ ) and with $L^{2}-f \rightarrow 0$, we preserve the throughput scaling as when we have full CSI. In figure (2), we plot the throughput versus the number of channel taps $L$ for various cluster sizes. This figure indicates how much variation (spread) the channel is able to withstand (using a certain cluster size) without suffering in the throughput. Figure (2) also suggests that we can treat a small number of subcarriers as a single subcarrier, and still maintain flat channel response. This is an interesting result which requires further investigation.

\section{CONCLUSIONS}

In this paper we investigate the throughput of wideband multi-antenna broadcast channels. We prove that for cluster size $\frac{N}{L \sqrt{\log K}}$, each user need only feedback the best SINR value at the center subcarrier of each cluster so that the transmitter can perform opportunistic beamforming by constructing $M$ beams on each subchannel and transmit to users with the best SINR values. This results in throughput scaling as when full CSI is available. Simulation results show that when cluster size is $\frac{N}{2 L}$, the loss in throughput is very small.

\section{REFERENCES}

[1] M. Sharif and B. Hassibi, "On the capacity of MIMO broadcast channel with partial side information," IEEE Trans. on Info. Theory, vol. 51, Feb. 2005.

[2] G. Caire and S. Shamai, "On the achievable throughput of a multi-antenna Gaussian broadcast channel," IEEE Trans. Inform., vol. 49, pp. 1691-1706, July 2003.

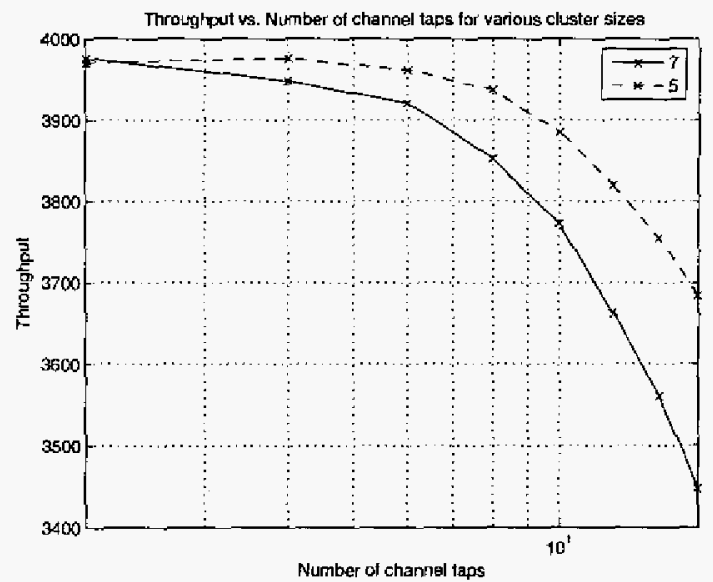

Fig. 2. Throughput vs. Number of channel taps for different Cluster sizes $(N=256, M=3, S N R=10$, Number of users $=1000$ ).

[3] P. Viswanath and D. N. Tse, "Sum capacity of the vector Gaussian broadcast channel and downlink-uplink duality," IEEE Trans. Inform., vol. 49, pp. 1912-192I, Aug. 2003.

[4] S. Vishwanath, N. Jindal, and A. Goldsmith, "Duality, achievable rates and sum rate capacity of Gaussian MIMO broadcast channel," IEEE Trans. Inform., vol. 49, no. 10, pp. 2658-2668, 2003.

[5] H. Weingarten, Y. Steinberg, and S. Shamai, "The capacity region of the gaussian MIMO broadcast channel," in Proc. of IEEE ISIT, 2004.

[6] M. Sharif and B. Hassibi, "Scaling laws of surn rate using time-sharing, DPC, and beamforming for MIMO broadeast channels," in Proc. of IEEE ISIT, 2004.

[7] P. Svedman, S. Wilson, L. Cimini, and B. Ottersten, "A simplified opportunistic feedback and scheduling scheme for OFDM," Proceedings IEEE Veh. Tech. Conference, May 2004.

[8] S. Sanayei, A. Nosratinia, and N. Aldhahir, "Opportunistic dynamic subchannel allocation in multiuser OFDM networks with limited feedback," IEEE Info. Theory Workshop, Oct. 2004.

[9] L. Toufi $k$ and R. Knopp, "Multiuser channel allocation algorithms achieving hard fairness," IEEE Global Communications Conference, vol. 1, Dec. 2004.

[10] D. Tse and P. Viswanath, Fundamentals of Wireless Communication. to be published by Cambridge University Press.

[11] J. Proakis, Digital Communications. McGraw-Hill Publishing Co., New York, 2001.

[12] R. Churchill and J. Brown, Complex Variables and Applications. McGraw-Hill Publishing Co., New York, 1990. 